This item was submitted to Loughborough's Research Repository by the author.

Items in Figshare are protected by copyright, with all rights reserved, unless otherwise indicated.

\title{
Multi-physics analysis of valve train systems: from system level to microscale interactions
}

PLEASE CITE THE PUBLISHED VERSION

PUBLISHER

Professional Engineering Publishing / @ IMECHE

VERSION

VoR (Version of Record)

LICENCE

CC BY-NC-ND 4.0

\section{REPOSITORY RECORD}

Teodorescu, M., M. Kushwaha, Homer Rahnejat, and Steve Rothberg. 2019. "Multi-physics Analysis of Valve Train Systems: From System Level to Microscale Interactions”. figshare. https://hdl.handle.net/2134/4726. 
This item was submitted to Loughborough's Institutional Repository (https://dspace.lboro.ac.uk/) by the author and is made available under the following Creative Commons Licence conditions.

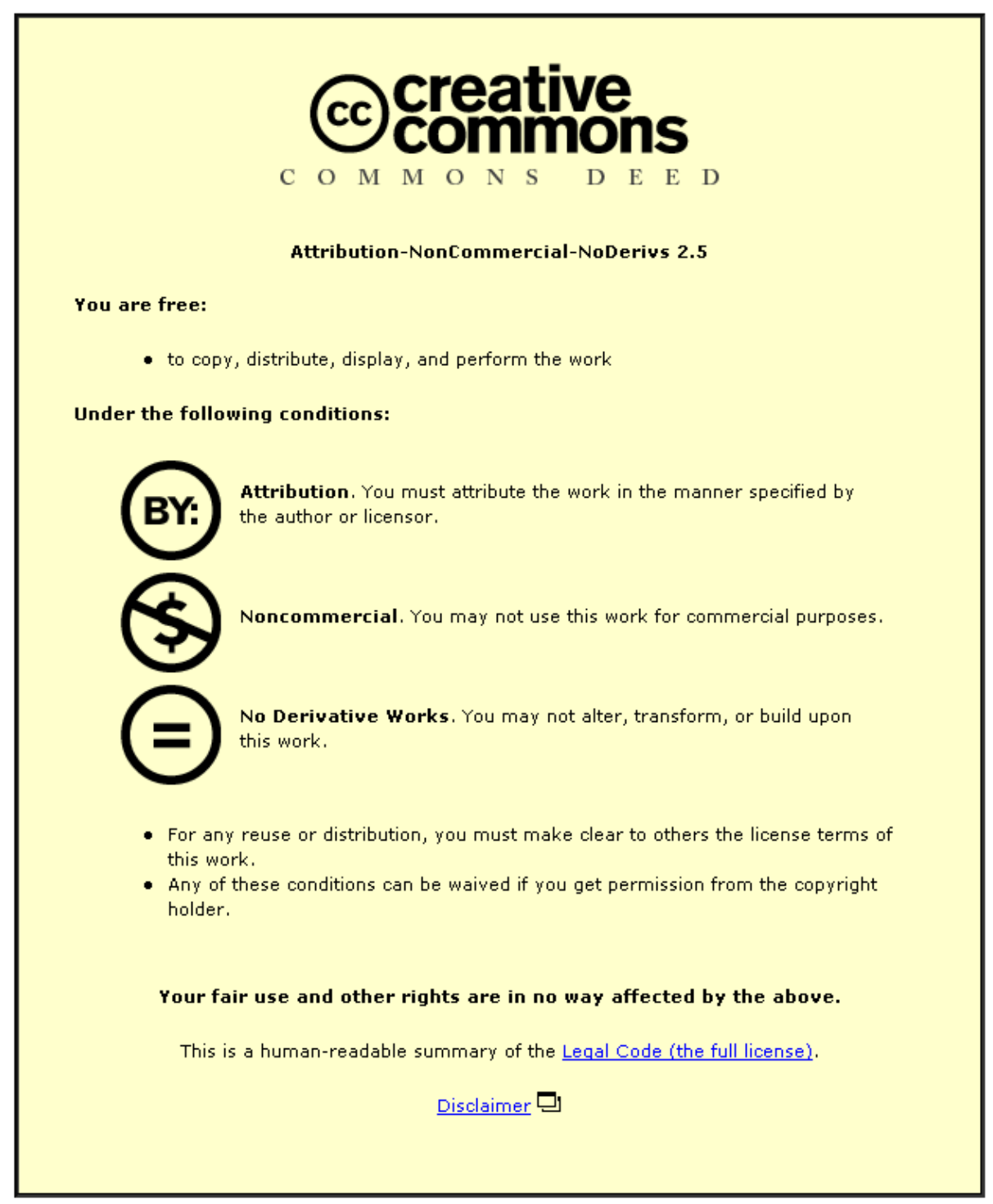

For the full text of this licence, please go to: http://creativecommons.org/licenses/by-nc-nd/2.5/ 


\title{
Multi-physics analysis of valve train systems: from system level to microscale interactions
}

\author{
M Teodorescu $^{1 *}$, M Kushwaha $^{2}$, H Rahnejat ${ }^{3}$, and S J Rothberg ${ }^{3}$ \\ ${ }^{1}$ School of Engineering and Mathematical Sciences, City University, London, UK \\ ${ }^{2}$ Ford Motor Company, Ford Engineering Centre, Essex, UK \\ ${ }^{3}$ Wolfson School of Mechanical and Manufacturing Engineering, Loughborough University, Leicestershire, UK
}

The manuscript was received on 3 May 2006 and was accepted after revision for publication on 8 January 2007.

DOI: $10.1243 / 14644193 J M B D 77$

\begin{abstract}
The paper highlights a holistic, integrated, and multi-disciplinary approach to design analysis of valve train systems, referred to as multi-physics. The analysis comprises various forms of physical phenomena and their interactions, including large displacement inertial dynamics, small amplitude oscillations due to system compliances, tribology, contact mechanics, and durability at the cam-tappet contact. Therefore, it also represents a multi-scale investigation, where the phenomena can be investigated at system level and referred back to underlying causes at subsystem or component level, in other words, implications of an event at microcosm can be ascertained on the overall system performance. This approach is often referred to in industry as down-cascading and up-cascading. The particular case reported here to outline the merits of this approach concerns a four-stroke single-cylinder engine. This promotes a system approach to engineering analysis for integrated noise, vibration, and harshness, durability and frictional assessment (efficiency). Experimental validation is provided with a motored test rig, using laser doppler vibrometry.
\end{abstract}

Keywords: valve train systems, noise, vibration, and harshness, frictional/tribological assessment, durability, multi-physics/multi-scale analysis

\section{INTRODUCTION}

The operation of a four-stroke internal combustion (IC) engine is heavily dependent on the behaviour of its valve train system. The concept is very simple and has been used long before the IC was even conceived, for example, in steam engines. Basically, a set of valves, which are closed during the highpressure region of the thermodynamic cycle open progressively during the gas exchange region of the cycle. However, the main design characteristics in valve train systems have evolved significantly since the early days. Given the low operational demand and over-design, most of the early engine components suffered malfunction because of gradual degradation in

* Corresponding author: School of Engineering and Mathematical Sciences, City University, Northampton Square, London EC1VOHB, UK.email:m.teodorescu@city.ac.uk the form of rust, rather than through mechanical failures. The development of later steam engines, and particularly, the then newly introduced IC engines, brought additional challenges, including the need to reduce mechanical and frictional losses, durability, and more recently noise and vibration refinement. Although engines have reduced in size and weight (use of lighter durable materials) the operating speeds and loads have actually increased by at least an order of magnitude. The IC engines have been evolved in line with the increasing demand put on efficiency with the additional requirement of reduced manufacturing costs. In recent years, the new generations of valve trains have become significantly lighter and subjected to much higher forces. Therefore, for every mechanism, the opening and the closing events are carefully designed to synchronize their mechanical cycle with the combustion cycle to achieve maximum efficiency. This has brought new challenges, which in the case of valve train systems translates into complex regimes of 
lubrication in cam-follower conjunctions (reduction of lubricant film, thus increased chance of wear), stressing of load bearing surfaces (greater tendency for inelastic subsurface deformation) and vibration because of component flexibility. Therefore, to be in line with these changes, a holistic and integrated approach is desired, which necessitates breakdown of the traditionally isolated engineering disciplines to promote the concept of first time right in product development in an ever increasing competitive market.

A typical valve train comprises a large number of contacting elements whose interactions are governed by a wide range of coupled phenomena. Consequently, to accurately predict their mechanical behaviour, a detailed transient dynamic model of the mechanism is an important prerequisite.

The ideal model should account for the transient interactions between several physical phenomena ranging from system level interactions to those at microscale. This approach is called multi-physics multi-scale. The approach requires integrated solution for all the interactive phenomena, such as Lagrangian dynamics for rigid body motions (of the order of few to several millimetres for valve motion), and Reynolds equation for lubricated conjunctions (for submicrometre thick films). For a complex system such as a valve train, this requires differential equations of motion for several parts, constraint functions for their assembly, Reynolds and elasticity equations for all the contacts. The result is a system of differential-algebraic equations, which must be solved simultaneously both in time and space domains.

The most loaded contact in the valve train mechanism is the cam-tappet conjunction. The sliding nature of this lubricated conjunction, together with a highly transient loading regime, renders this contact a major cause of valve train mechanical inefficiency. Without the protective effect of a lubricant film formed in the contact, the applied load together with vibrations can cause significant frictional losses and in extreme cases wear and scuffing of contacting surfaces. A multi-physics model is reported here, which incorporates all the aforementioned physical phenomena.

The validity of a mathematical model can only be accepted if at least some of its predictions are verified against experimental measurements. In order to understand the effect of certain physical phenomenon, a fundamental test (fully independent of the method of analysis) is required. The experience gained, is then integrated back into the comprehensive framework of the whole system. The opposite extreme is the use of a fully instrumented engine test $[1,2]$. Although much closer to reality, the resulting measurements should be very carefully analysed in order to eliminate the influence of other additional interactions. Because of the combustion process, the results are usually affected by noise, and great care should be taken in applying corrective methods. Therefore, the optimal approach may be the use of a test rig, in which an engine is motorized, rather than fired [3-6]. The advantage of this technique is that most of the additional noise is eliminated and the resulting signal still preserves the interactions between the key physical elements of the system.

\section{THE MULTI-PHYSICS VALVE TRAIN MODEL}

\subsection{System dynamics}

A constrained inertial dynamics model is required, which should incorporate the elastic behaviour of the various valve train elements. Each component contributes to the system dynamics through its stiffness and damping properties, and special care is taken while modelling the contacting/impacting conditions. The general framework is built on the method described by Teodorescu et al. [7]. Figure 1 is a schematic representation of the equivalent valve train mechanism as a two-mass system model with three degrees of freedom. Each lumped mass element $\left(m_{1}, m_{2}\right)$ is considered to be rigid and represents the mass of several components of the valve train system. Mass $m_{1}$ represents the tappet, the push-rod, and the proportion of the rocker arm in translation on the push-rod side of the assembly. Mass $m_{2}$ represents the valve, effective mass of the valve spring (being one-third of its actual mass) and the contribution of the mass of the rocker arm on the valve side. The connecting springs and dampers are considered to be massless. The contacts in the mechanism are shown in Fig. 1 by two horizontal parallel lines. The possible frictional losses are also introduced in the model through inclusion of appropriate coefficients of friction for the contacting solids in relative motion.

The governing equations of motion are obtained for each element of the lumped masses, using the Newton-Euler method, and solved together in a time marching scheme. The general format for equation of motion is

$$
\begin{gathered}
m_{i} \ddot{z}_{i}+c_{e_{i}}\left(\dot{z}_{i}-\dot{z}_{i-1}\right)+k_{e_{i}}\left(z_{i}-z_{i-1}\right)-k_{e_{i+1}}\left(z_{i+1}-z_{i}\right) \\
-c_{e_{i+1}}\left(\dot{z}_{i+1}-\dot{z}_{i}\right)+F_{i}=0 \quad i=1,2
\end{gathered}
$$

where, the friction between various components of the valve train, as well as the possible additional forces are 


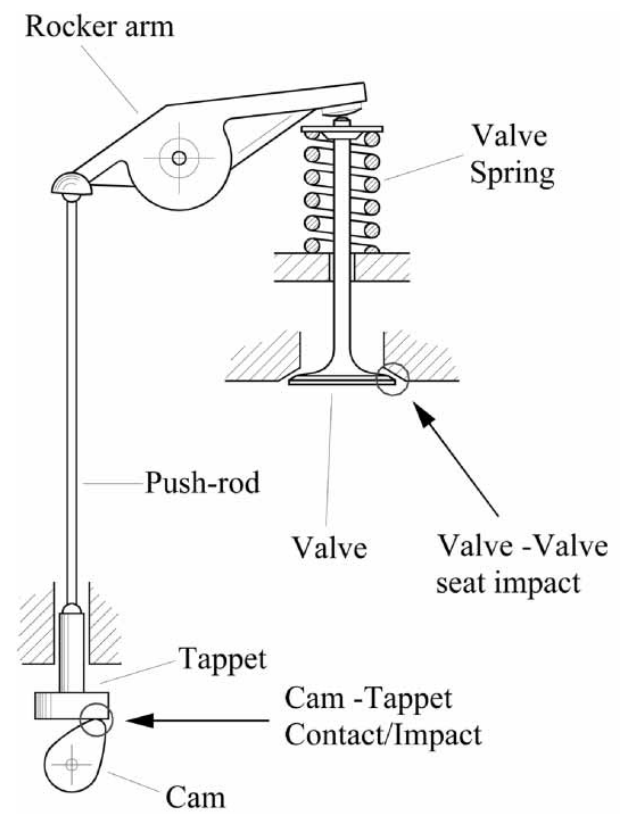

a) Schematic view of the mechanism

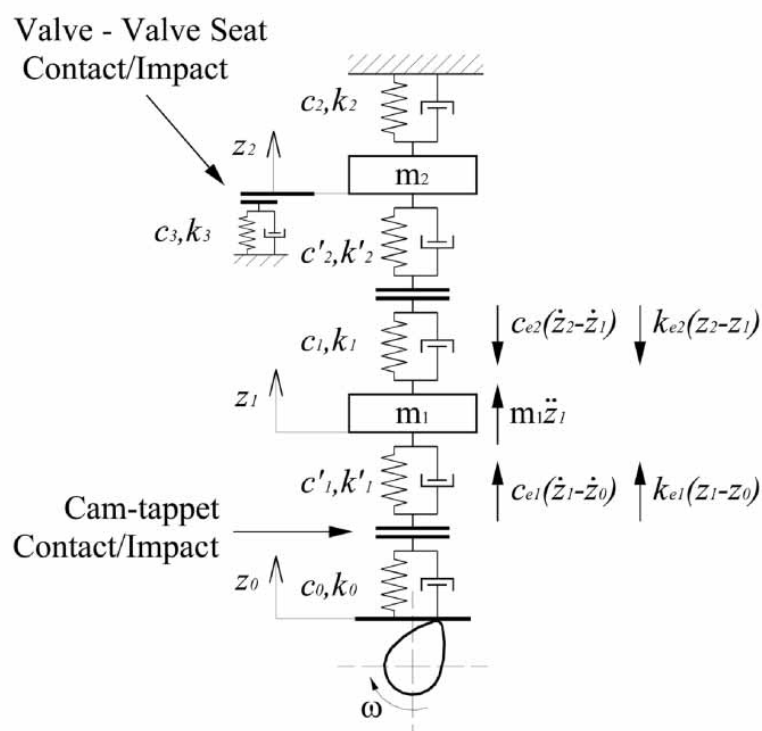

b) Equivalent model

Fig. 1 Dynamic model for the valve train system

given by the term $F_{i}$, and

$$
\begin{aligned}
& \begin{cases}k_{e_{i}}=\frac{k_{i}^{\prime} \cdot k_{i-1}}{k_{i}^{\prime}+k_{i-1}}, c_{e_{i}}=\frac{c_{1}^{\prime} \cdot c_{i-1}}{c_{i}^{\prime}+c_{i-1}} & \text { contact } \\
k_{e_{i}}, c_{e_{i}}=0 & \text { loss of contact } \\
& i=1,2\end{cases} \\
& k_{e_{3}}=k_{2}, c_{e_{3}}=c_{3}
\end{aligned}
$$

The contact force between the cam and the tappet is required in equation (1) for the mass $m_{1}$. This is effectively the integrated contact pressure distribution in the cam-tappet conjunction, which means that for proper analysis transient consideration of this contact is necessary.

\subsection{Cam-tappet conjunction}

The conjunction between the cam and the tappet accounts for the major portion of frictional losses in the valve train system and should, therefore, be included in the system model. This is a finite line conjunction, which can be represented at any instant of time by the contact of an equivalent roller near a semi-infinite elastic half-space. Not withstanding the stress concentrations at the edges of the cam to tappet contact in the direction of cam width, the pressure gradient along the length of the equivalent roller may be considered small compared with that in the direction of lubricant entrainment: $\partial P / \partial y \ll \partial P / \partial x$. Therefore, as a first approximation, an infinite line contact solution may be undertaken, with the repercussion that the minimum film thickness is considered to be in the vicinity of the exit boundary, and not that in the exit region and to the sides of the contact, as would be under physical conditions. Furthermore, any side leakage of lubricant is considered to be negligible. Thus, solution for this conjunction may be obtained using the following one-dimensional form of Reynolds' equation

$$
\frac{\partial}{\partial x}\left(\frac{\rho h^{3}}{\eta} \frac{\partial P}{\partial x}\right)=12\left[u \frac{\partial(\rho h)}{\partial x}+\frac{\partial(\rho h)}{\partial t}\right]
$$

where, the contact is considered as an infinite line configuration with $x$ denoting the direction of entraining motion of the lubricant. Ideally, the cam-tappet contact should be considered as a finite line contact and the complex tappet dynamics [8] taken into consideration.

The elastic film shape is given by

$$
h=h_{0}+\delta
$$

where, $h_{0}$ is the instantaneous rigid separation, including the profile of the cam at any location in the $x$-direction against the flat tappet, and $\delta$ is the instantaneous local deflection at any location in the contact, obtained by solution of the contact elasticity problem as

$$
\delta(x)=\frac{1}{\pi E^{\prime}} \int_{x^{*}=x_{\text {inlet }}}^{x^{*}=x_{\text {outlet }}} P\left(x^{*}\right) D\left(x, x^{*}\right) \mathrm{d} x^{*}
$$

where, $E^{\prime}=2 /\left(1-v_{1}^{2} / E_{1}\right)+\left(1-v_{2}^{2} / E_{2}\right)$ is the reduced Young's modulus of elasticity of contacting pairs. 
Where, $\delta$ is obtained at any location in the contact because of all pressure elements within the instantaneous contact pressure distribution and $D$ is the influence coefficient matrix [9].

Now, clearly the extent of pressure distribution from inlet to outlet must be determined. The outlet boundary condition is thus required, and Reynolds' boundary condition is implemented as

$$
\begin{aligned}
\left.P\right|_{x_{\text {inlet }}} & =0 \text { at } x=-\infty \text { (fully flooded), and }\left.P\right|_{x_{\text {outlet }}} \\
& =\left.\frac{\partial P}{\partial x}\right|_{x_{\text {outlet }}}=0
\end{aligned}
$$

Now to solve Reynolds' equation, one needs the rheological state of the lubricant $(\eta, \rho)$. Viscosity variation with pressure is taken into account using Roelands' equation [10]

$$
\eta=\eta_{0} \exp \left[\ln \eta_{0}+9.67\right]\left[\left(1+5.1 \times 10^{-9} P_{h}\right)^{Z}-1\right]
$$

where $Z=\alpha /\left(5.1 \times 10^{-9}\left[\ln \eta_{0}+9.67\right]\right)$.

Density variation with pressure is given by Dowson and Higginson [11]

$$
\rho=\rho_{0}[1+0.6 P /(1+1.7 P)]
$$

Now the lubricant reaction acting on mass $m_{1}$, as already described is found as

$$
F_{i}=\int_{x_{\text {inlet }}}^{x_{\text {outlet }}} P \mathrm{~d} x \text { for } i=1
$$

Kinematics of the contact $(u, \partial h / \partial t)$ are essential for the solution of Reynolds' equation. The speed of entraining motion of the lubricant is the average velocity of the contacting surfaces in the $x$-direction. Disregarding tappet spin [12]

$$
u=\left[R_{\mathrm{c}}+\frac{1}{\omega^{2}} \frac{\partial^{2} s}{\partial t^{2}}\right] \frac{\omega}{2}
$$

The elastic squeeze film velocity is $\partial h / \partial t=\partial h_{0} / \partial t+$ $\partial \delta / \partial t$ (equation (4)). This shows that the approach/ separation of contacting surfaces takes place by a combination of rigid body motion (the first term, when negative indicates approach of surfaces) and their local rate of deformation (when positive indicates an emerging gap by local deformation of the surfaces). This is easily computed under transient conditions as (first-order approximation) $\partial h / \partial t=1 / \Delta t\left(h^{j}-h^{j-1}\right)$, where $j$ denotes the time step and $\Delta t$ the analysis time step size. Therefore, in transient analysis $\partial h / \partial t$ represents the historical variation in local contact conditions at any location $x$, and can be termed as film memory.

\subsection{Determination of friction}

Friction in cam-tappet contact arises because of viscous and boundary contributions. Where a coherent lubricant film is assured, the contribution to friction is because of viscous action under sliding condition alone. However, thin films are encountered at inlet reversal positions (where the cessation of entraining motion takes place, prior to and after the cam nose). Therefore, interruptions in a coherent film may occur, depending on the composite roughness of the contacting surfaces in relative motion. A parameter, $\gamma=h / \psi$ (ratio of film thickness to the composite roughness of the contiguous surfaces), is used to ascertain any boundary contributions, which occur because of asperity interactions. This occurs when $\gamma<3$. The composite surface roughness of cam and tappet surfaces for the contact considered is measured to be $0.2 \mu \mathrm{m}$.

The elemental areas in the contact region are given as

$$
\mathrm{d} A=B \mathrm{~d} x
$$

For each element, the asperity contact area and the load carried by the asperities are computed, based upon the model proposed by Greenwood and Tripp [13] as

$$
\begin{aligned}
\mathrm{d} A_{\mathrm{a}} & =\pi^{2}(\zeta \beta \psi)^{2} F_{2}(\gamma) \mathrm{d} A \\
\mathrm{~d} F_{\mathrm{a}} & =0.75 \pi(\zeta \beta \psi)^{2} \sqrt{\frac{\gamma}{\beta}} E^{\prime} F_{5 / 2}(\gamma) \mathrm{d} A
\end{aligned}
$$

The two statistical functions $F_{2}$ and $F_{5 / 2}$ are determined using the formulation proposed in $[\mathbf{1 3}]$ as

$$
F_{n}(\gamma)=\frac{1}{\sqrt{2 \pi}} \int_{\lambda}^{\infty}(s-\gamma)^{n} e^{-s^{2} / 2} \mathrm{~d} s
$$

In order to speed-up the numerical predictions, the method proposed by Teodorescu et al. [14] for a line contact is adopted here, and a fifth degree polynomial is fitted to each of the corresponding statistical functions.

The total friction force, acting on an element $\mathrm{d} A$ is computed as the sum of the viscous and boundary friction components as

$$
F=\int\left(\mathrm{d} F_{\mathrm{b}}+\mathrm{d} F_{\mathrm{v}}\right) \mathrm{d} x
$$

The boundary friction force results from the shearing of a very thin film (down to several layers of molecules), which prevails in the contact between the asperity tips during their contact. The friction force is computed as described in references [13] and [14]. The non-Newtonian shear stress $\tau_{\mathrm{E}}$ is given as a function of the normal load components [15]. Consequently, the 
boundary friction force, acting on an element can be expressed as

$$
\mathrm{d} F_{\mathrm{b}}=\tau_{0} \mathrm{~d} A_{a}+m \mathrm{~d} F_{\mathrm{a}}
$$

The general form for the viscous friction force only takes into account the shear of the lubricant film, which is trapped between the sliding surfaces outside the asperity contact areas. Thus

$$
\mathrm{d} F_{\mathrm{v}}=\left\{\begin{aligned}
& \frac{\eta u}{h}\left(\mathrm{~d} A-\mathrm{d} A_{\mathrm{a}}\right) \longrightarrow \frac{\eta u}{h} \leqslant \tau_{\mathrm{E}} \approx 2 \mathrm{MPa} \\
&\left(\tau_{E}+0.8 \frac{\mathrm{d} F-\mathrm{d} F_{a}}{\mathrm{~d} A}\right)\left(\mathrm{d} A-\mathrm{d} A_{\mathrm{a}}\right) \\
& \longrightarrow \frac{\eta u}{h} \geqslant \tau_{\mathrm{E}} \approx 2 \mathrm{MPa}
\end{aligned}\right.
$$

\subsection{Subsurface stress field}

Load bearing contacts represent the most critical components in many machines and mechanisms. An ideal load bearing conjunction would possess sufficient compliance to undergo local deformation under load. This enhances the formation of a protective lubricant film, while having an adequate hardness, to avoid excessive wear because of surface asperity interactions of the contiguous solids. Excessive contact pressures can also cause inelastic deformation due to subsurface stress field, exceeding the elastic limit, and causing fatigue spalls. Cam-tappet contact is the most loaded conjunction in IC engines, and any multi-physics analysis should incorporate proper contact mechanics of this conjunction.

Harmonic decomposition techniques have always presented an opportunity to be used in such applications, although their primary use has been in the determination of global deformation of elastic solids, such as in bending and buckling of plates and shells [16]. The small dimensions of non-conforming contacts provide an interval of very short length. This means that a pressure distribution, acting upon the contact area, must be approximated by a harmonic series of significant number of orders. An additional consideration is that the local nature of deformation is preserved below the natural modal behaviour of the contacting solids (see the Hertzian impact theory), thus restricting the upper limit of the harmonic series. This combination of restrictions, together with the averaging nature of the fast Fourier analysis has presumably been the reason for the late take-up of this approach as an alternative to the traditional method of predicting contact behaviour.

Figure 2 shows the contact conditions as well as the pressure distribution. This practical problem corresponds to the plane strain condition, as pointed out

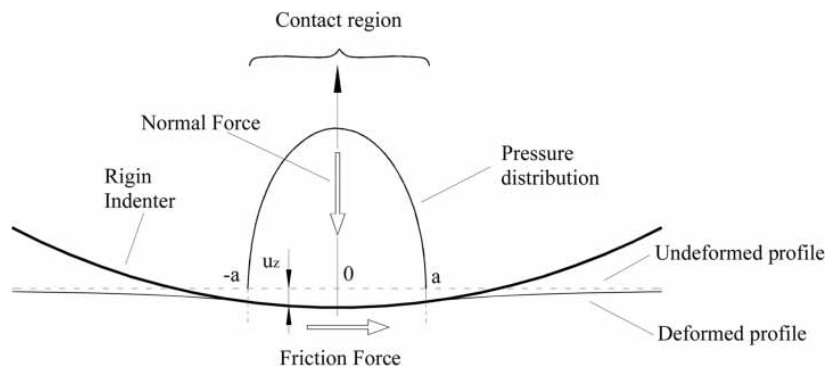

Fig. 2 Transverse pressure distribution on the contact

by Hertz [17]. To achieve a realistic prediction, both the contact load as well as the contact friction should be considered. Since the pressure distribution over the contact footprint is half an ellipse, it is also assumed that shear also has a similar distribution.

Both the contact pressure and surface shear distributions can be decomposed into Fourier series over a chosen interval, larger than the footprint width in this case. The size of this interval $2 L$ should be large enough to avoid excessive influence because of edge effects, thus

$$
\left\{\begin{array}{l}
P=0.5 P_{0}+\sum_{k=1}^{\infty} P_{k}\left(\frac{2 \pi x}{\lambda_{k}-\varphi_{k}}\right) \\
\tau=0.5 \tau_{0}+\sum_{k=1}^{\infty} \tau_{k}\left(\frac{2 \pi x}{\lambda_{k}-\psi_{k}}\right)
\end{array}\right.
$$

where

$$
\alpha_{k}=2 \pi / \lambda_{k} \quad \text { and } \quad \lambda_{k}=L / k
$$

The stress field can be computed for each harmonic component (wavelength) separately and the final prediction is obtained using the superposition principle. Teodorescu et al. $[\mathbf{1 8}]$ have shown that the final subsurface stress tensor can be expressed as

$$
\left\{\begin{aligned}
k \sigma_{x x} & \operatorname{sech} \alpha_{k} y \\
= & {\left[P_{k} \cos \left(\alpha_{k} x-\varphi_{k}\right)\left(\alpha_{k} y-1\right)\right.} \\
& \left.+\tau_{k} \sin \left(\alpha_{k} x-\psi_{k}\right)\left(\alpha_{k} y-2\right)\right]\left(\tanh \alpha_{k} y-1\right) \\
{ }_{k} \sigma_{y y} & \operatorname{sech} \alpha_{k} y \\
= & {\left[-P_{k} \cos \left(\alpha_{k} x-\varphi_{k}\right)\left(\alpha_{k} y+1\right)\right.} \\
& \left.+\tau_{k} \sin \left(\alpha_{k} x-\psi_{k}\right) \alpha_{k} y\right]\left(\tanh \alpha_{k} y-1\right) \\
{ }_{k} \sigma_{x y} & \operatorname{sech} \alpha_{k} y \\
= & {\left[-P_{k} \sin \left(\alpha_{k} x-\varphi_{k}\right) \alpha_{k} y\right.} \\
& \left.+\tau_{k} \cos \left(\alpha_{k} x-\psi_{k}\right)\left(\alpha_{k} y-1\right)\right]\left(\tanh \alpha_{k} y-1\right)
\end{aligned}\right.
$$

Note that most of the modern cam and tappets are protected with wear resistant coatings. The general 
method to deal with such cases takes into account the effect of thin- or thick-layered bonded solids [19].

The pressure distribution is decomposed into a number of harmonic components and a line of constant pressure. The effect of this latter term on the contact stresses is taken into account here.

For any location $x, y$ under the surface the stresses can be evaluated as [20]

$$
\left\{\begin{aligned}
0 \sigma_{x x}= & -\frac{P_{0}}{2 \pi}\left[2\left(\theta_{1}-\theta_{2}\right)+\left(\sin 2 \theta_{1}-\sin 2 \theta_{2}\right)\right] \\
& +\frac{\tau_{0}}{2 \pi}\left[4 \ln \left(\frac{r_{1}}{r_{2}}\right)-\left(\cos 2 \theta_{1}-\cos 2 \theta_{2}\right)\right] \\
{ }_{0} \sigma_{y y}= & -\frac{P_{0}}{2 \pi}\left[2\left(\theta_{1}-\theta_{2}\right)-\left(\sin 2 \theta_{1}-\sin 2 \theta_{2}\right)\right] \\
& +\frac{\tau_{0}}{2 \pi}\left(\cos 2 \theta_{1}-\cos 2 \theta_{2}\right) \\
{ }_{0} \sigma_{x y}= & \frac{P_{0}}{2 \pi}\left(\cos 2 \theta_{1}-\cos 2 \theta_{2}\right)-\frac{\tau_{0}}{2 \pi}\left[2\left(\theta_{1}-\theta_{2}\right)\right. \\
& \left.+\left(\sin 2 \theta_{1}-\sin 2 \theta_{2}\right)\right]
\end{aligned}\right.
$$

where

$$
r_{1,2}=\sqrt{(x \mp L / 2)^{2}+y^{2}}, \quad \tan \theta_{1,2}=y /(x \mp L / 2)
$$

\section{EXPERIMENTAL SET-UP}

In the current work, a 5-Hp single-cylinder Honda IC engine was powered by a variable speed electric motor. The goal was to validate the dynamics of the complete valve train model, as well as the cam profile, by monitoring the movements of the tappet in the test rig. Since the dynamics of the valve train system is of primary interest, the piston and the rest of engine auxiliaries were removed. Several openings were made in the engine block to ensure access for measurements to be carried out. Figure 3 depicts an electric motor (1) and the test engine (2).

Monitoring of the motion of valve train components at high acceleration can be achieved with a variety of methods. Norton et al. [21] have analysed the vibration characteristics of a valve train system, providing a critique of the various available methods. The cylinder head was mounted on a separate fixture, and one of the intake valves was instrumented. Teodorescu et al. [14] installed a miniature accelerometer on the valve seat of a fired engine and monitored the acceleration over a large range of engine speeds. One of the common conclusions made is that ideally the method of measurement should not interfere with the valve train geometry. Therefore, for the current study, a non-contacting method was employed. A laser Doppler vibrometer (LDV) was used to measure the tappet lift velocity. The method consists of a

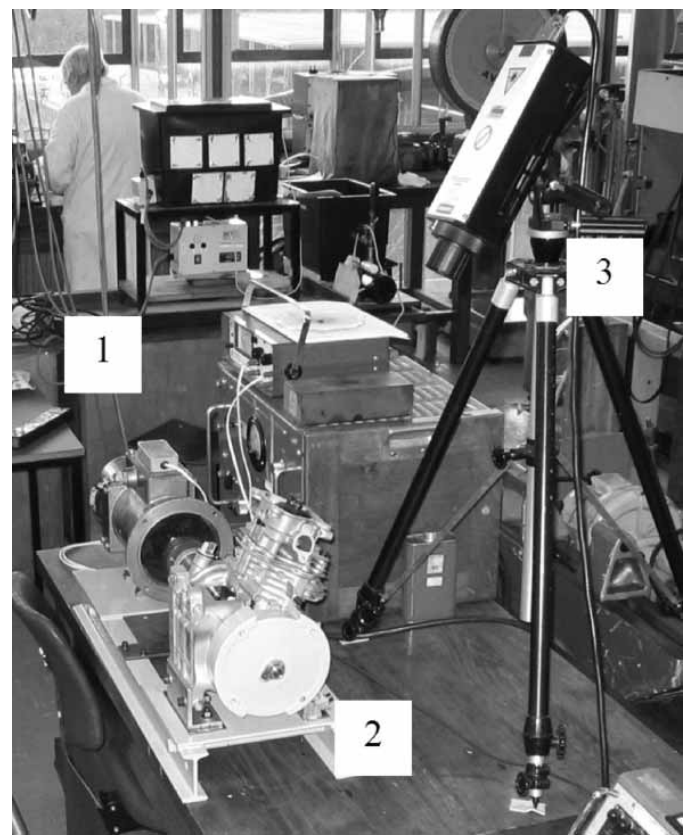

Fig. 3 The experimental set-up

laser source (point 3 in Fig. 3), which transmits a single beam towards the tappet. The upper side of the tappet is covered with a special reflective paper to reduce the chance of beam scatter. Figure 4 shows the principle of operation of an LDV. To reduce the noise and to minimize the measurement errors, the beam was carefully centred orthogonal to the upper side of the tappet at all times. An attribute of cam-tappet contact is that the cam is designed to approach the tappet with a predefined eccentricity. During the event

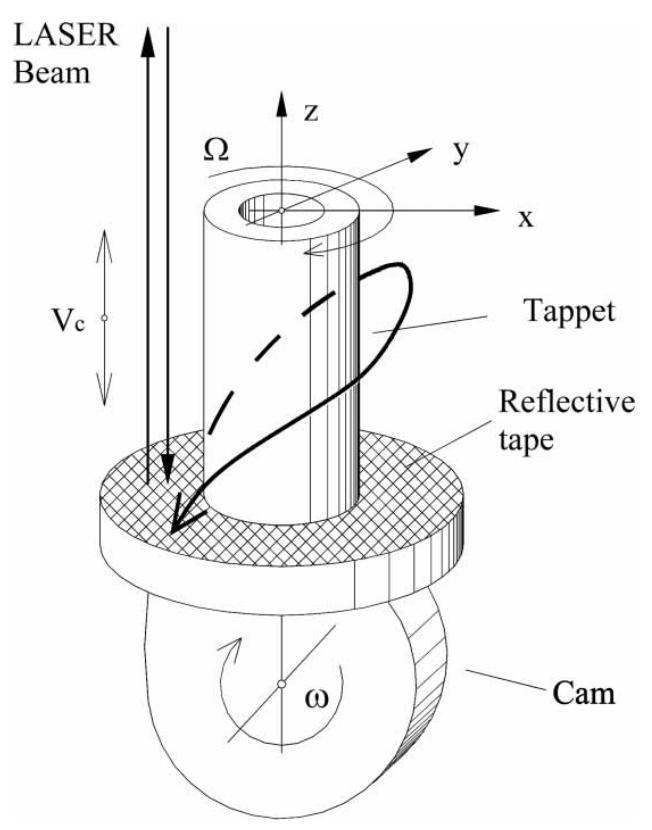

Fig. 4 Principle of use of the LDV 
cycle, this misalignment generates a moment along the vertical axis of the tappet, and consequently, the tappet tends to spin. Teodorescu et al. [22] have investigated the tappet spin phenomenon. This, together with the vertical motion of the tappet can alter the LDV measurements and result in an error. However, this shortcoming can be eliminated if the laser beam is directed orthogonally to the reflective surface of the target.

\section{RESULTS AND DISCUSSION}

\subsection{Experimental validation}

A suitably designed mechanism should ensure required valve timing and sealing. This can be achieved through numerous methods. The most commonly used method is to introduce a small clearance in the mechanism, ensuring correct valve closure durations with no additional applied force. A downside to this approach is that at the start of the event cycle, the clearance is suddenly taken-up by the cam lift, with all the mechanism components coming into contact, thereby, resulting in an undesired impact. Therefore,

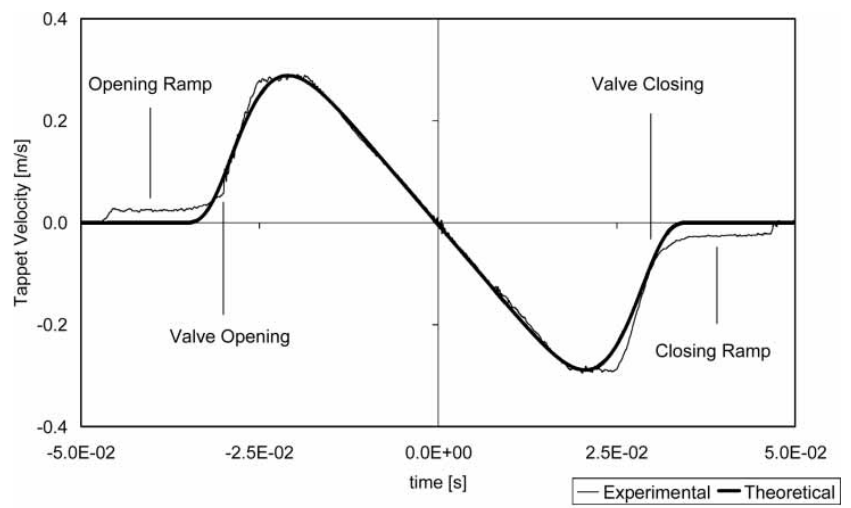

Measurement and prediction at $630 \mathrm{rpm}$

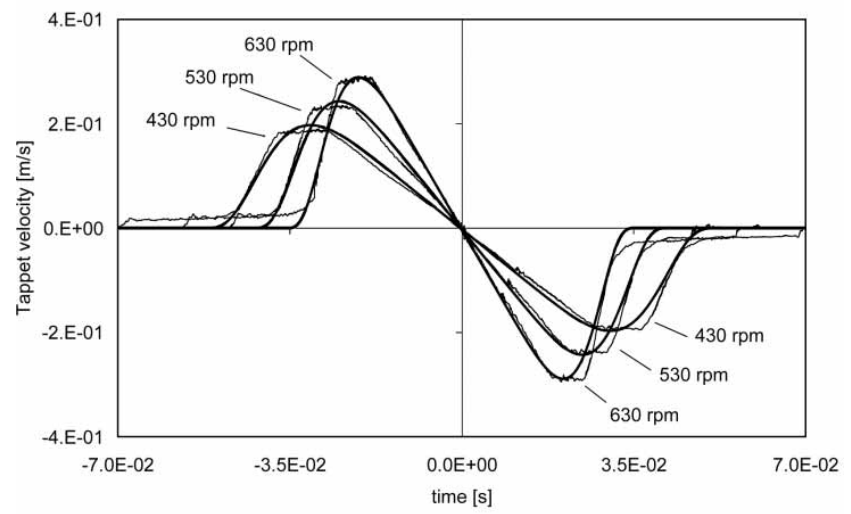

Measurement and prediction at three engine speeds

Fig. 5 Comparison between the experimental and theoretical tappet velocity

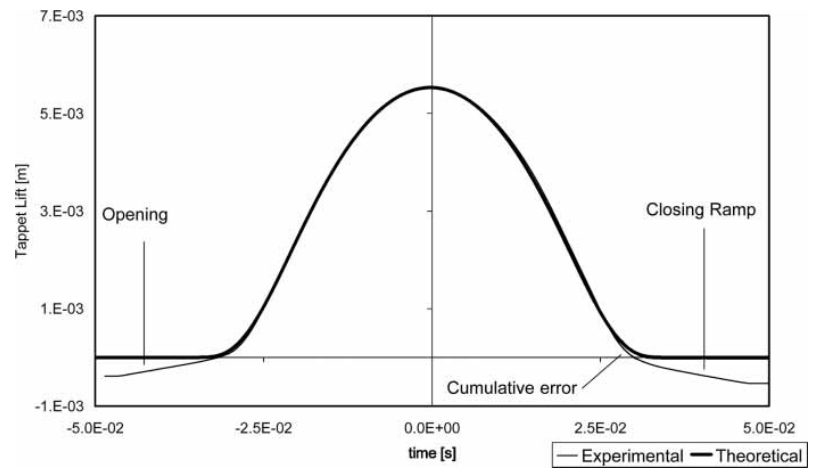

Fig. 6 Experimental and theoretical cam lifts

the engine life may be affected by the high impact forces. A simple method of overcoming this problem is to introduce a ramp at the beginning and at the end of the cam profile. The geometrical profile of such a ramp has been investigated in some detail by Chen [23]. Because of the vertical orientation of the experimental set-up, the cam and tappet are likely to be in contact during the part of the cycle, when the valve is not actuated. Figure 5(a) shows the measured tappet velocity, together with the predictions provided by the model. Two constant velocity ramps, one before the opening part of the event and the other after closure can be observed. Since the mechanism is not loaded during these ramps, the velocity remains constant and consequently, there is no acceleration. Thus, the ramps are of little interest in the combined durability and noise, vibration, and harshness assessment, and thus are not included in the model.

The experiment was repeated for several engine speeds to verify consistent behaviour. Figure 5(b) shows the tappet velocity for three different engine speeds, together with the corresponding model predictions. The stretch of the curve at lower speeds is due to a longer time required for the mechanism to describe the cam profile.

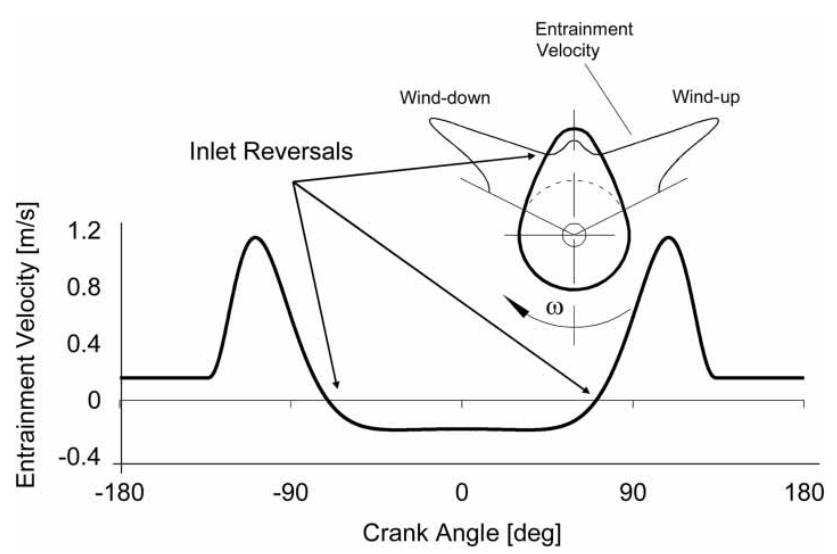

Fig. 7 Lubricant entrainment velocity 


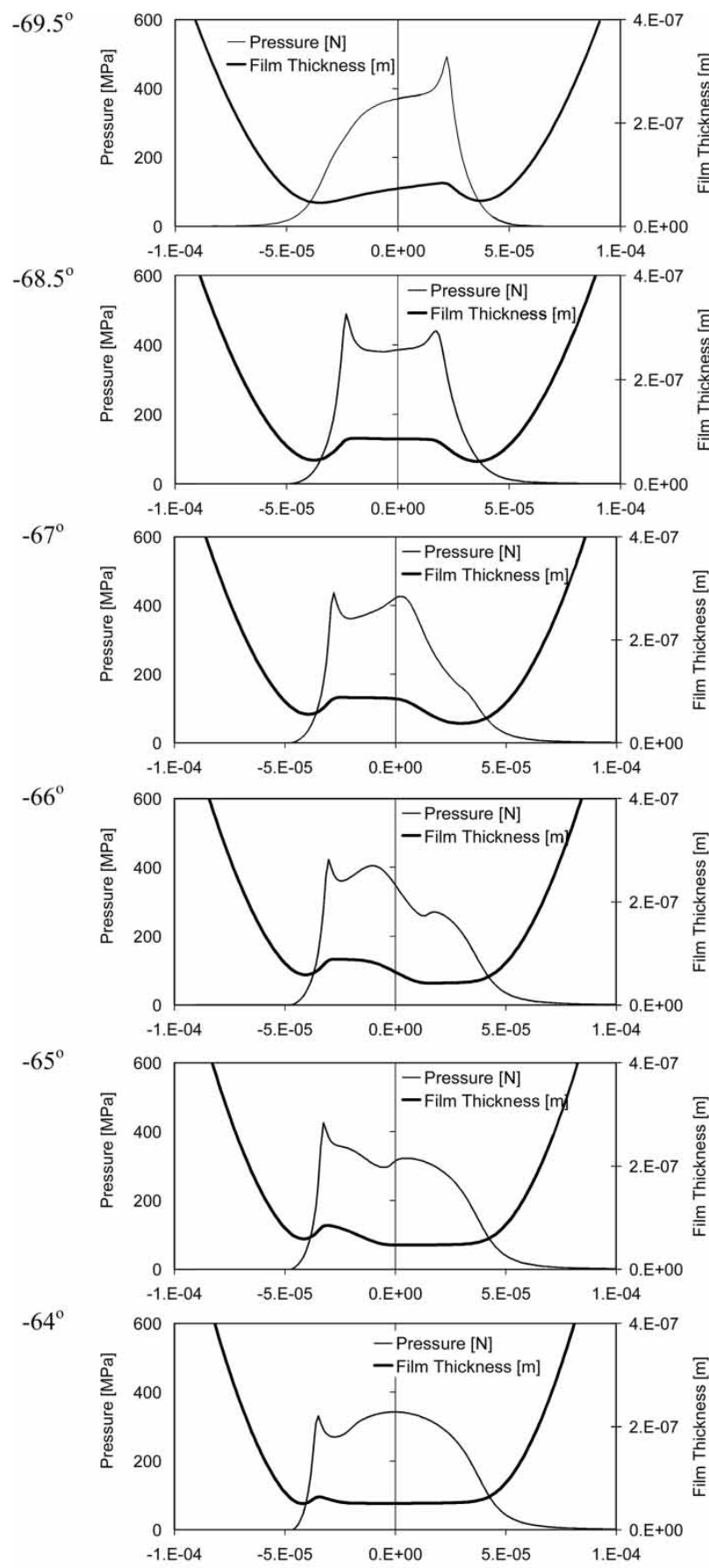

(a)
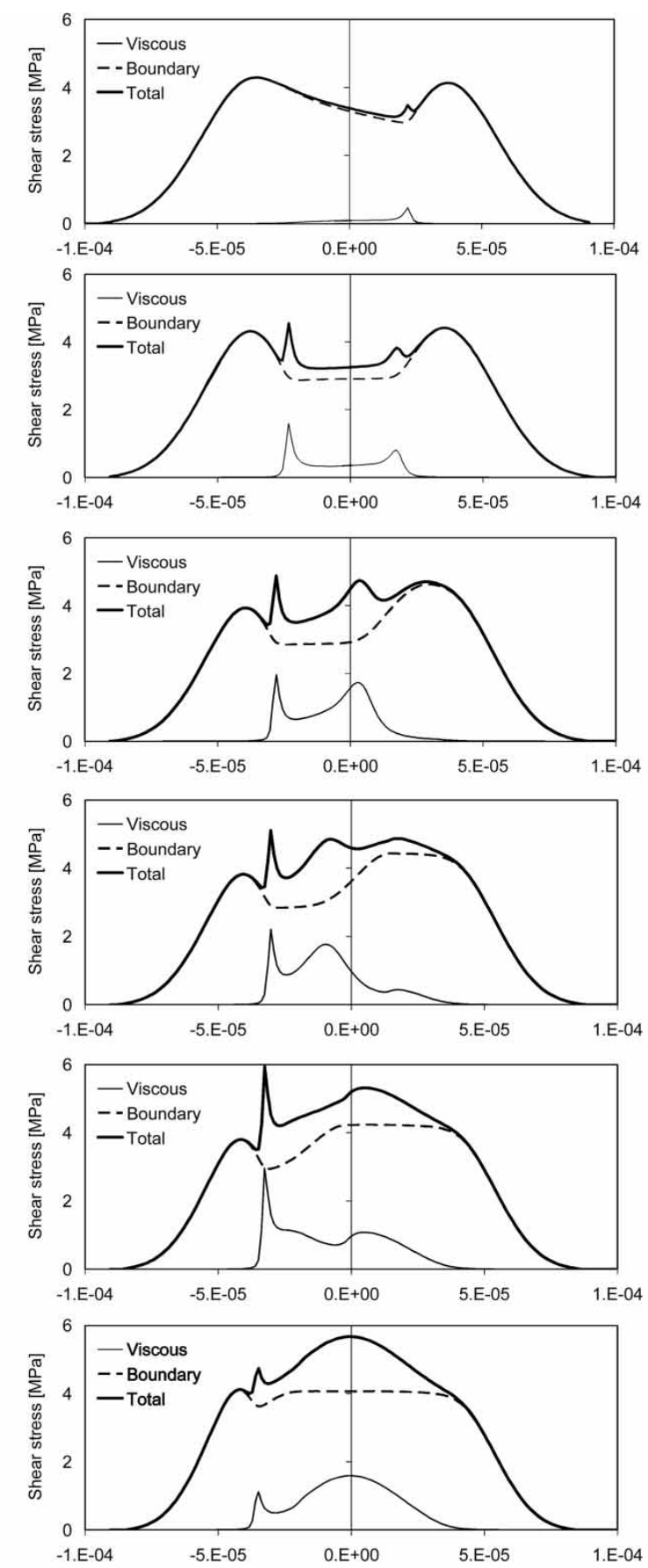

(b)

Fig. 8 Transient pressure and shear distributions and film shape through the inlet reversal during the wind-up process

The predicted values for tappet velocity contain lower noise levels than those obtained experimentally. This is partly random, due to measurement error, and partly repetitive across the engine speed spectrum, due to surface irregularities, not accounted for in the model. The simulation of the dynamic behaviour of the mechanism requires an accurate prediction of the tappet acceleration. However, by differentiating the experimental signal, the noise levels are extensively amplified. The integration of the tappet velocity curve with respect to time yields the tappet lift as shown in Fig. 6.

It should be noted that the integration process also acts as a smoothing algorithm. Therefore, the cumulative error over the whole cam event results in a small skew of the curve at the end of the closure event. 
A high-order polynomial cam is utilized in the mathematical model. The polynomial function for the cam was achieved by employing curve fitting techniques to the experimentally obtained cam lift data $[\mathbf{2}, \mathbf{2 3}]$.

\subsection{Down-cascading from system level to microscale contact interactions}

Now that good agreement is obtained between the predictions and the experimental data, the model results can be used to study many important design and performance aspects, where measurements are difficult to undertake, such as from cam-tappet interface.

Figure 7 shows the lubricant entrainment velocity for a cam cycle. At the beginning and at the end of the cam event, the cam-tappet contact is on the cam flanks, where the speed of entraining motion and instantaneous radius of curvature of the cam attain their highest values. Consequently, the oil film is relatively thick and the regime of lubrication is usually hydrodynamic, with low friction, and thus not of great interest. However, around $\pm 70^{\circ}$ from the position of cam nose the speed of entraining motion ceases and changes direction. These positions are known as inlet reversal regions because of a change in the direction of lubricant flow into the contact. At these locations the supply of fresh lubricant by wedge action ceases and an oil film survives purely due to the squeeze film action [8]. In the extreme cases of high load and low engine speeds, a mixed regime of lubrication is most likely in these locations, where boundary friction would contribute to the contact frictional behaviour. The cam nose region usually suffers the highest contact load, but the nearly constant speed of entraining motion ensures a continuous supply of lubricant, and the regime of lubrication is predominantly piezoviscous elastic (i.e. elastohydrodynamic lubrication (EHL)). It is interesting to ascertain whether these experiential findings are borne out by the predictions of the multi-physics model.

Figure 8(a) shows a series of pressure distributions and the corresponding lubricant film thickness, as the cam traverses through the wind-up region (inlet reversal point at around $-70^{\circ}$ ). Note that the inlet trail of the pressure distribution (on the right-hand side of the figure) gradually changes direction (to the left-hand side). The solitary pressure spike, typical of elastohydrodynamic (EHD) pressure distributions, migrates from the initial outlet position (on the left-hand side) to the final outlet location. In the process of migration, gradually a pressure spike emerges at the opposite end of the contact as the initial spike begins to diminish in magnitude. This is an important phenomenon, as the pressure spike inhibits the flow of lubricant in the region directly beneath it, thus depleting the film and increasing the chance of direct surface interactions (see later). Correspondingly, a cave (dimple) appears in the elastic film shape. This is because of the elastic squeeze effect. This cave moves within the contact, which indicates the movement of a pressure wave through the contact at inlet reversal points. This phenomenon tends to generate a higher film thickness during these parts of the cycle, where the cessation of entraining motion would normally be expected to cause the depletion of the oil film. The elastic squeeze film is dominated by the local distortion of surfaces, being a much quicker elastic phenomenon than the slow viscous response of the lubricant being pushed out of the contact by rigid approach, and is referred to above as film memory. It is an unintentional fortunate event that happens to be also engrained in nature, such as in natural mammalian joints [24]. The results here also corroborate the conclusions of Kushwaha and Rahnejat [25] that squeeze caving phenomenon is really a by-product of kinematics of the contact.

Figure 8(b) shows the corresponding shear stress distribution for those cases shown in Fig. 8(a). These indicate the prevalence of mixed regime of lubrication through the wind-up inlet reversal event, being the contribution due to boundary lubrication. It is also clear that the total shear behaviour puts the contact condition firmly into non-Newtonian for the most of the contact as $\tau>\tau_{\mathrm{E}}$. However, the viscous contribution remains largely in the Newtonian regime, except in the vicinity of the migrating pressure spike in the latter parts of the wind-up process. In fact, its contribution is almost insignificant prior to the inlet reversal, and during the lift event. As the cave depth initially increases the shear stress is reduced and as the inlet is completely reversed (the culmination of the wind-up process), both the viscous and boundary contributions increase accordingly. This shows that squeeze caving event is in fact quite a favourable tribological event on its own accord, which has been somewhat underrated.

The same conditions also occur at around $+70^{\circ}$, where the wind-down process (subsequent inlet

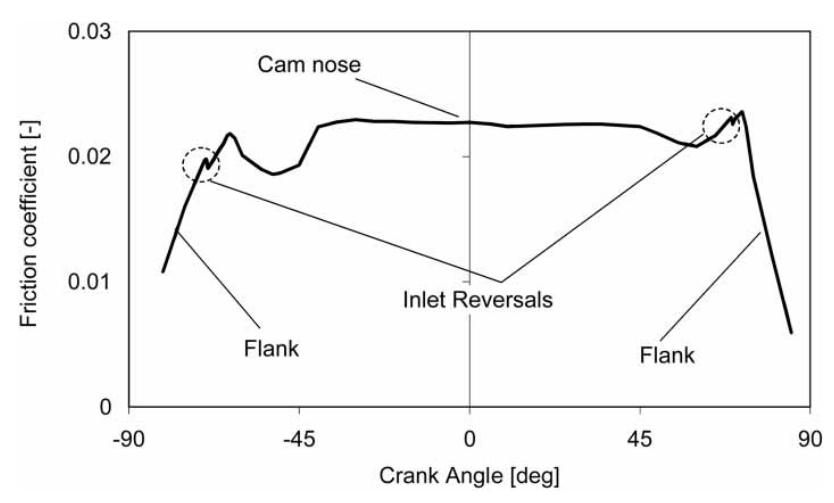

Fig. 9 Cyclic variations of coefficient of friction 
(a) without shear

(b)

$-69.5^{\circ}$

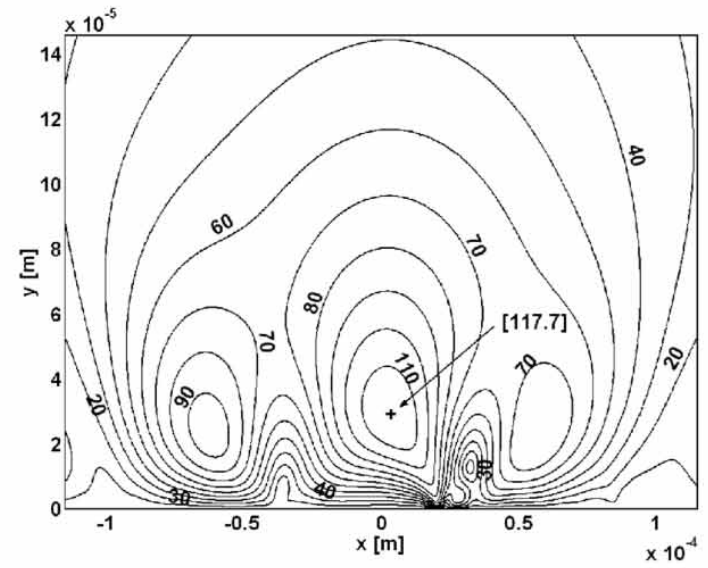

(c)

$-68.5^{\circ}$

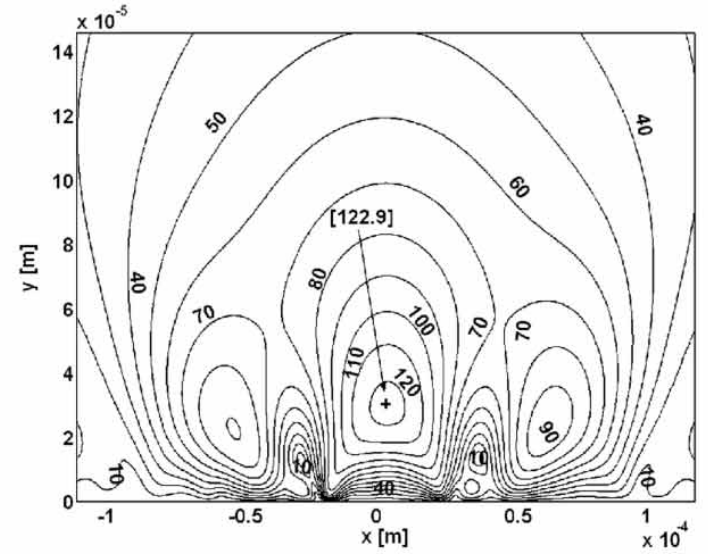

(d) $\quad-64^{\circ}$

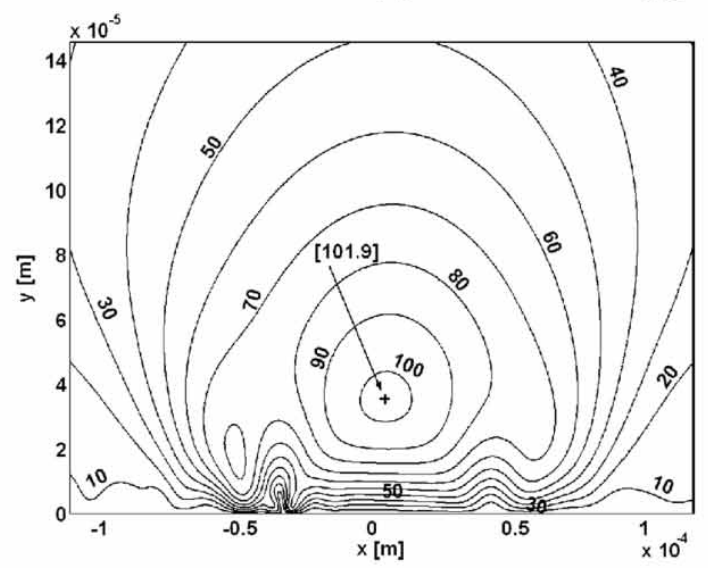

Maximum shear stress field
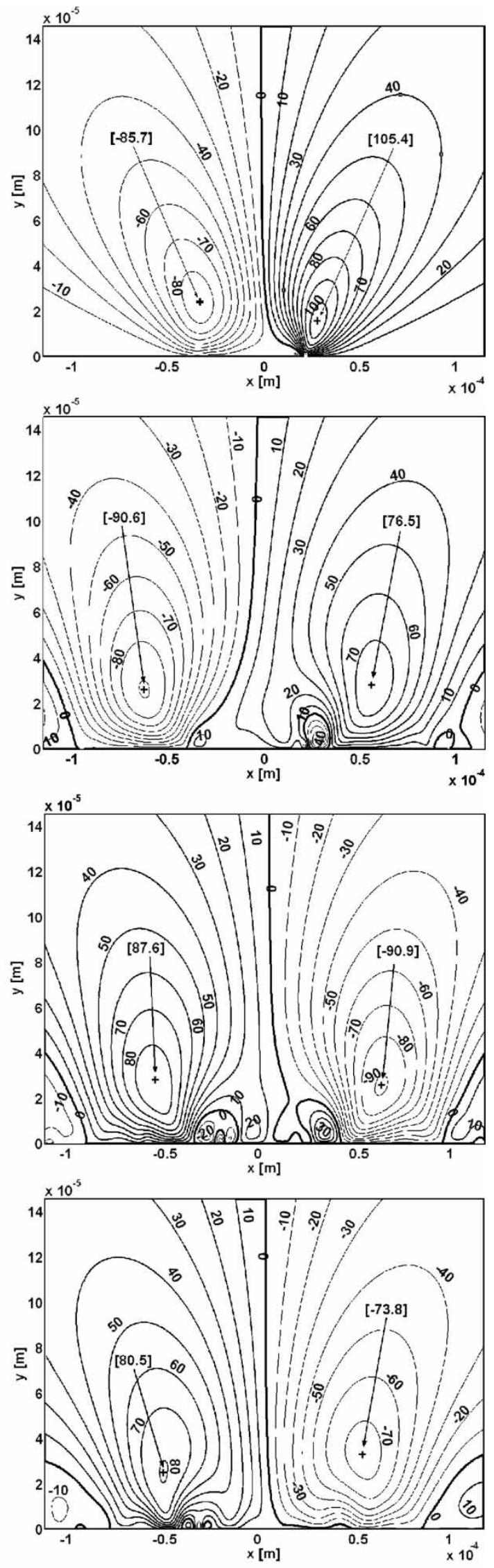

Orthogonal shear stress field

Fig. 10 Subsurface stress fields 
reversal) takes place, but lower pressure spikes and shallower dimple formation are noted.

As the regime of lubrication alters from the cam flank-to-tappet contact through the inlet reversal points and onto the cam nose, as well as the rheological behaviour of fluid from dominantly Newtonian to non-Newtonian, the coefficient of friction changes accordingly. Figure 9 shows that the coefficient of friction falls rapidly on the flank contacts as hydrodynamic conditions are attained, with mainly Newtonian viscous friction. Between the reversal points and onto the cam nose, the coefficient of friction hardly alters and has its maximum value, with the prevalent mixed regime of lubrication, dominated by boundary friction. At and in the vicinity of inlet reversals due to low speed of entraining motion boundary contribution dominates, as already shown in Fig. 8(b), but the formation of the squeeze cave actually mitigates against the undesired effect of friction. This is a major fundamental find, suggesting that application of soft coating layers in these regions of cam may lead to a reduction in frictional losses. Investigations of this type, in more detail, may pave the way to localized economical application of appropriate coatings (modulus of elasticity and layer thickness), rather than the current experimental ad hoc approach. A migrating pressure spike in a softer conjunction can lead to a greater depth of squeeze cave, a phenomenon already demonstrated in Fig. 8(a).

The pressure spike sets up localized subsurface stress field of its own, superimposed upon the overall subsurface stress tensor, increasing both the magnitude of stresses and reducing the depth beneath the surface that such stresses attain their highest values, thus exists a greater chance for fatigue spalling. Figure 10(a) shows this asymmetry in the maximum shear stress field, indicated by the isocline at $120 \mathrm{MPa}$, tending towards the surface of the tappet (i.e. at $y=0$ ). This absolute maximum island of shear stress follows the Hertzian theory at $0.3 P_{\mathrm{H}}$, where $P_{\mathrm{H}}$ is the maximum Hertzian pressure (the primary pressure peak in the EHL pressure distribution, at the top left-hand pressure distribution of Fig. 8(a)). Under elastostatic Hertzian condition, the contact semi-half-width in the $x$-direction would be $0.03 \mathrm{~mm}$. The depth at which the maximum shear stress should occur would be 78 per cent of this semi-half-width (or $0.0234 \mathrm{~mm}$ ), which is predicted to be $0.024 \mathrm{~mm}$ in the case of Fig. 10(a). This shows very good agreement with the classical theory. The shift along the $x$-axis is due to the presence of the EHL pressure spike. The asymmetry due to pressure spike is better observed in the subsurface stress tensor component $\sigma_{x y}$. This is the orthogonal reversing shear stress, which is of a cyclic nature as can be seen at the top left-hand inset in Fig. 10. Note that the isoclines in the vicinity of the pressure spike pack very closely and approach the surface.
This shows that sufficiently high pressures can lead to fatigue.

However, the contact is subjected not only to applied pressures, but also to shear as already discussed. Thus, Fig. 10(a) is only informative. The correct predictions for the contact condition are those in Fig. 10(b), where the effect of shear leads to an increase in magnitude of shear stresses near the surface as well as further asymmetry in the subsurface stress tensor. Further islands of stress are formed due to the migration of the pressure pip, with its localized field propagating from $x>0$ to $x<0$ with the squeeze cave (Figs 10(a) to (d)).

Figure 11 provides better visualization of the formation of the squeeze cave and its migration at the inlet reversal during the wind-up process. Figure 11(a) shows the depth of the dimple emerging during the onset of contact transition from cam flank (inlet reversal). The step increase in the film thickness indicates the emergence of the dimple (Fig. 8(a)). The depth increases gradually (and also migrates along the $x$-direction (Fig. 11(c))) until the dominance of elastic squeeze ceases and entraining motion of the lubricant commences, indicated by the sharp drop in the dimple depth. In the corresponding Fig. 11(b), a negative value for the squeeze velocity indicates rigid approach. Thus, to the left of the indicated demarcation line (on the cam flank just prior to the inlet reversal) a small approach of surfaces is noted. At the event, a sharp rise in $\partial h / \partial t>0$ indicates the dominance of the elastic nature of squeeze velocity (i.e. $|\partial \delta / \partial t| \gg\left|\partial h_{0} / \partial t\right| \therefore$ $\partial h / \partial t>0)$, creating the squeeze cave. Thereupon, the effect of squeeze diminishes $(\partial h / \partial t \approx 0)$ and the depth of the cave remains more or less unchanged

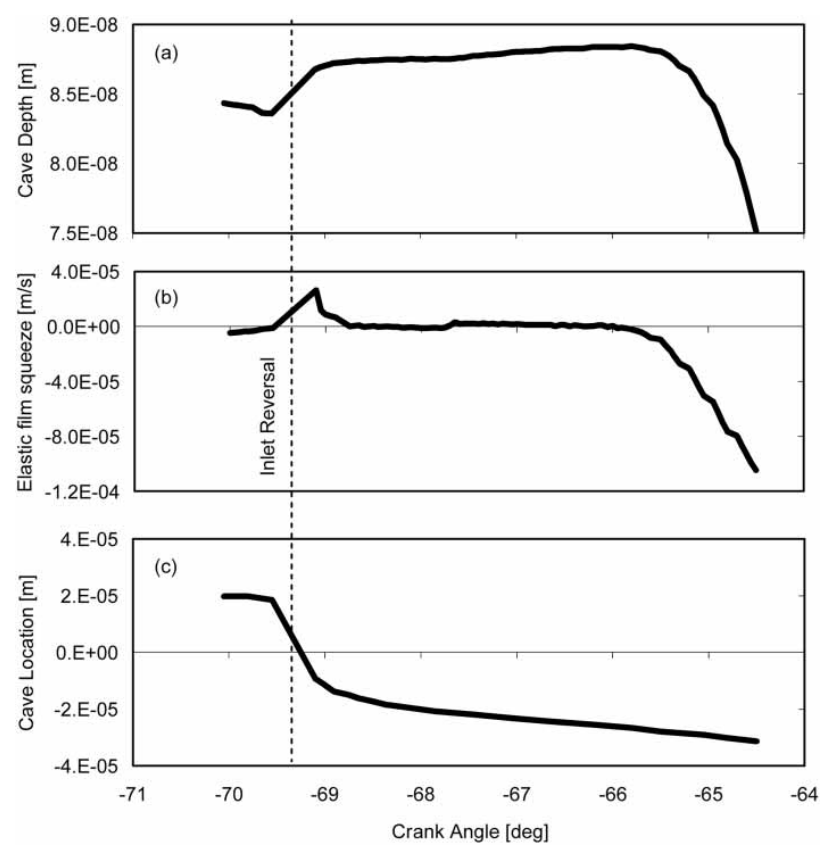

Fig. 11 Kinematics of squeeze cave phenomenon 
(Fig. 11(a)), but is pushed along the contact (Fig. 11(c)) with the reinstatement of entraining motion. This is the sign of migration of the pressure spike across the contact (Fig. 8(a)), with the eventual large approach of the surfaces $(\partial h / \partial t>0)$ to smoothen the cave and attain the familiar EHL pressure distribution shape dominated by entraining motion (Fig. 8(a), the inset at the bottom left-hand corner). The predictions here show that squeeze caving is essentially governed by contact kinematics, as correctly observed by Kushwaha and Rahnejat [25] and need not be explained in terms of a thermal wedge as has been invariably suggested as a cause, which is in fact clearly an effect.

\section{CONCLUSIONS}

The multi-physics model provides an integrated tribodynamic analysis, coupled with the evaluation of subsurface stress field. The model results at the system level agree with experimental measurements. With down-cascading, even to microscale not only additional information of a practical nature is found, but also some fundamental aspects of physics of motion can be ascertained. Further physical phenomena such as heat generation due to work done should be added in future work, which would affect lubricant rheology, thus the contact conditions and consequently the system behaviour.

\section{REFERENCES}

1 Ball, W. F., Jackson, N. S., Pilley, A. D., and Porter, B. C. The friction of a 1.6 litre automotive engine-gasoline and diesel, SAE paper 860418, 1986.

2 Teodorescu, M., Taraza, D., Henein, N. A., and Bryzik, W. Experimental analysis of dynamics and friction in valve train systems. Trans. SAE, J. Engine, 2002, 1027-1038.

3 Crane, M. E. and Meyer, R. A process to predict friction in an automotive valve train. SAE paper 901728, 1990 .

4 Pieprzak, J. M., Willermet, P. A., and Dailey, D. P. Experimental evaluation of tappet/bore and cam/tappet friction for a direct acting bucket tappet valve train. SAE paper 902086, 1990.

5 Van Helden, A. K., Van der Meer, R. J., Van Staaden, J. J., and Van Geldern, E. Dynamic friction in cam/tappet lubrication. SAE paper 850441, 1985.

6 Yutaro, W., Mitsushiro, S., Yoshito, E., Toshiro, H., and Tatsumi, K. Studies on friction characteristics of reciprocating engines. SAE paper 952471, 1995.

7 Teodorescu, M., Rahnejat, H., and Rothberg, S. Elastomulti-body dynamics of valve train systems with spring surge effect and coil clash. In Multi-body dynamics: monitoring and simulation techniques III (Eds H. Rahnejat and S. Rothberg), 2004, pp. 273-284 (Professional Engineering Publishing, IMechE, London).
8 Kushwaha, M. and Rahnejat, H. Transient elastohydrodynamic lubrication of finite line conjunction of cam to follower concentrated contact. J. Phys. D, Appl. Phys., 2002, 35 (3), 2872-2890.

9 Hamrock, B. J. Fundamentals of fluid film lubrication, 1994 (McGraw-Hill International Editions, New York).

10 Roelands, C. J. A. Correlation aspects of viscositytemperature-pressure relationship of lubricating oils. PhD Thesis, Delft University of Technology, The Netherlands, 1996.

11 Dowson, D. and Higginson, G. R. Elasto-hydrodynamic lubrication, 1966 (Pergamon Press Ltd, Oxford).

12 Teodorescu, M., Kushwaha, M., Rahnejat, H., and Taraza, D. Elastodynamic transient analysis of fourcylinder valve train system with camshaft flexibility. Proc. IMechE, Part K: J. Multi-body Dynamics, 219(K1), 2005, 13-25.

13 Greenwood, J. A. and Tripp, J. H. The contact of two nominally flat rough surfaces. Proc. Instn Mech. Engrs, 1970-71, 185(48/71), 625-633.

14 Teodorescu, M., Taraza, D., Henein, N. A., and Bryzik, W. Simplified elasto-hydrodynamic friction model of the cam - tappet contact. Trans. SAE, J. Engine., 2003, 12711283.

15 Evans, C. R. and Johnson, K. L. The rheological properties of elastohydrodynamic lubricants. Proc. Instn Mech. Engrs, Part C: J. Mechanical Engineering Science, 1986, 200 (C5), 303-312.

16 Timoshenko, S. P. and Goodier, J. N. Theory of elasticity, 1951 (McGraw-Hill, New York).

17 Hertz, H. On the contact of elastic solids. J. Reine Angew. Math., 1881, 92, 156-157.

18 Teodorescu, M., Rahnejat, H., and Gohar, R. Harmonic analysis to determine contact characteristics of concentrated counterformal contacts. In Proceedings of the 7th Biennial ASME Conference on Engineering Systems Design and Analysis, Manchester, UK, 19-22 July 2004.

19 Teodorescu, M. and Rahnejat, H. Mathematical model for elastic layered contacts with direct application on a cam-tappet conjunction within a multi-physics framework. In Applied mathematical modelling (Elsevier Science).

20 Johnson, K. L. Contact mechanics, 1985 (Cambridge University Press, Cambridge).

21 Norton, R. L., Stene, R. L., Westbrook, J. I., and Eovaldi, D. Analyzing vibrations in an engine valve train. SAE technical paper 980570, 1998.

22 Teodorescu, M. and Taraza, D. Numerical prediction and experimental investigation of cam - to flat tappet lubricated contact with tappet spin. Proc. Instn Mech. Engrs, Part K: J. Multi-body Dynamics, 2004, 218(K3), 133-142.

23 Chen, J. S. Dynamic analysis of a 3D finger follower valve train system coupled with flexible camshafts. SAE technical paper 2000.

24 Dowson, D. and Jin, Z. M. Micro-elastohydrodynamic lubrication of synovial joints. Eng. Med., 1986, 15(2), 63-65.

25 Kushwaha, M. and Rahnejat, H. Transient concentrated finite line roller-to-race contact under combined entraining, tilting and squeeze film motion. J. Phys. D, Appl. Phys., 2002, 37, 2018-2034. 


\section{APPENDIX}

\section{Notations}

A contact area

$A_{\mathrm{a}} \quad$ asperity contact area

$B \quad$ cam width

$c_{i} \quad$ generic damping coefficient

$E_{1}, E_{2} \quad$ elastic moduli for cam and follower materials

$E^{\prime} \quad$ reduced modulus of elasticity: $E^{\prime}=$ $2 /\left\{\left(1-v_{1}^{2}\right) / E_{1}+\left(1-v_{2}^{2}\right) / E_{2}\right\}$

$F \quad$ total friction force

$F_{\mathrm{a}} \quad$ force carried by asperities

$F_{\mathrm{b}} \quad$ boundary friction force

$F_{\mathrm{i}} \quad$ applied forces

$F_{\mathrm{n}} \quad$ statistical functions

$F_{\mathrm{v}} \quad$ viscous friction force

$h \quad$ elastic film shape

$h_{0} \quad$ rigid separation

$k$ harmonic order

$k_{i} \quad$ generic stiffness

$L \quad$ Fourier decomposition interval

$m \quad$ pressure coefficient of the boundary shear strength

$m_{1} \quad$ equivalent mass of push-rod and tappet

$m_{2} \quad$ equivalent mass of valve assembly

$P \quad$ pressure

$P_{\mathrm{H}} \quad$ maximum Hertzian pressure

$P_{k} \quad$ amplitude of the $k$ th harmonic of the applied pressure $k=0 \rightarrow N$

$r_{1}, r_{2} \quad$ radial extent of the subsurface compressive stress field

$R_{\mathrm{c}} \quad$ instantaneous radius of curvature of cam cam lift

time

speed of entraining motion of the lubricant

coordinate along the contact surface coordinate into the depth of the contacting solids

rigid body motions

pressure viscosity index

piezo-viscosity index

$=2 k \pi / L$

asperity radius of curvature

film thickness parameter

local elastic deflection

time step size

surface density of asperity peaks

dynamic viscosity of the lubricant

atmospheric dynamic viscosity of the lubricant

$\theta_{1}, \theta_{2} \quad$ circumferential extent of the subsurface compressive stress field

$\lambda_{k} \quad=L / K$ wavelength for the $k$ th harmonic

$v_{1}, v_{2} \quad$ Poisson's ratio for cam and follower materials

$\rho$

atmospheric bulk density of the lubricant

${ }_{k} \sigma_{i j} \quad k$ th component of the stress tensor, where $i, j \in\{x, y\}$

$\tau_{k} \quad$ amplitude of the $k$ th harmonic of the applied shear stress $k=0 \rightarrow N$

Eyring shear stress of the oil composite surface roughness camshaft angular velocity 